\title{
The potential of protein-based nanocages for imaging and drug delivery
}

\section{Fabio Corsi ${ }^{a}$, Serena Mazzucchelli ${ }^{{ }^{*}}$}

a Dipartimento di Scienze Biomediche e Cliniche "L. Sacco", Università di Milano, Ospedale L. Sacco, via G. B. Grassi, 74, 20157 Milano, Italy

*serena.mazzucchelli@gmail.com; Tel.+3902390440450

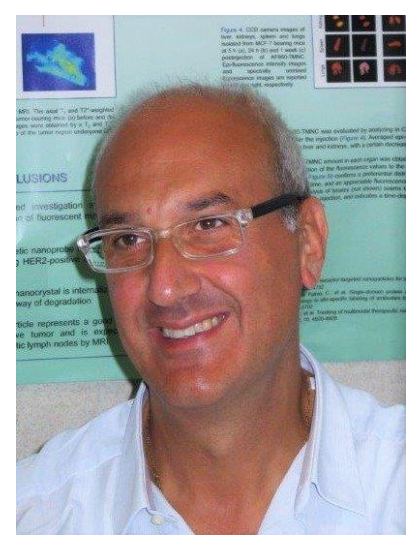

Fabio Corsi, Prof. MD

Dipartimento di Scienze Biomediche e Cliniche "L. Sacco", Università di Milano, Ospedale L. Sacco, via G. B. Grassi, 74, 20157 Milano, Italy

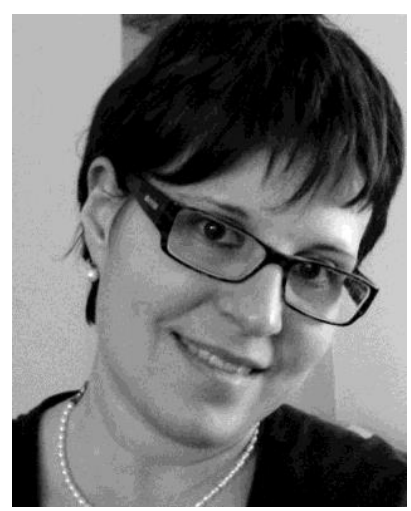

Mazzucchelli Serena, PhD

Dipartimento di Scienze Biomediche e Cliniche "L. Sacco", Università di Milano, Ospedale L. Sacco, via G. B. Grassi, 74, 20157 Milano, Italy 


\section{Acknowledgements}

The research was supported by the Fondazione Regionale per la Ricerca Biomedica (NANODRUG platform project). FRRB supported S.M

Key words: nanotechnology, protein based-nanocages, Emerging technologies

Nanoparticles have been deeply studied as carrier for imaging and drug delivery, but their clinical application still remains an unrealized dream. Nanodrugs have the potential to address some important concerns related to bioavailability, tissue penetration, circulation time, toxicity and protection from degradation/sequestration [1] since their size, surface charge and geometry may affect pharmacokinetic profiles [2]. Indeed, nanoparticles could be exploited to allow of the crossing of drugs through physiological barriers, to reach targeted sites, and to increase drugs half life and efficacy while reducing doses. Several nanoparticles, such as metal oxides, quantum dots, carbon nanotubes, liposomes and polymers, have been investigated at the preclinical level [3]. However, only few of them have been clinically approved since toxicity, immunogenicity and sequestration by liver reticulo-endothelial system still remain the main hindrances to translation to the clinic. [4]. Therefore, although great clinical appealing, nanotechnology are still poorly being translated into clinical practice [5].

In this scenario, protein-based nanocages represent a particular class of nanomaterials that may address many of these concerns [6]. The charm of protein-based nanotechnology relies on the fact that proteins often have minimal toxicity and immunogenicity, and can be easily degraded by the organism once they have carried out their function [7]. Protein caged nanoparticles are constituted by self-assembling protein subunits generally arranged in a hollow structure, which displays symmetrical repetitive elements; caged architectures could be constituted by unique or multiple proteins that are the building block of the nanoparticles $[1,7]$. Protein monomers could be produced in living hosts (i.e. E. coli, plants, mammalian cells, yeast and baculoviruses) and genetically 
modified inserting surface functionalities by DNA-recombinant technology, allowing a fine control of surface charge, ligand display, nanoparticles stability and active molecule encapsulation [7]. Besides, the nanocage building blocks are arranged to form a shell, which possess three distinct contact point: the interior, the interface and the exterior. These regions can be chemically or genetically engineered to insert pharmaceuticals and/or probes or to shape various and more ordered functionalities [1]. The simultaneous presence of these three region of interface in one single cage allows the contemporary display of different capabilities, enabling the realization of multifunctional nanoparticles, such as nano-probes for imaging detection of lesions combined with drug delivery devices for therapeutic purposes. Unlike other protein-based nanomaterials, protein nanocages possess some other unique features very appealing from the clinical point of view: 1) their uniform cage allow the precise control of the amount of encapsulated drugs/probes, which is crucial in define drug dosage; 2) avoidance of macromolecular aggregation of the nanoparticle; 3) stability in physiological environment as a result of their protein nature, with subsequent increased circulation time, protection of the cargo molecule from degradation and improved bioavailability [1]. Moreover, protein nanoparticles are resistant to a wide range of $\mathrm{pH}$ and temperature, so they are compatible with the chemical environment required for modifications [8]. Furthermore their capability to disassemble the quaternary structure in response of a precise stimulus is particularly useful to drugs and/or probes encapsulation [9]. Finally, due to their reduced size, protein cages are able to escape from the reticulo-endothelial system increasing their accumulation to the target site [10].

These features are strongly appealing from the clinical point of view since the development of a smart drug delivery system is a primary challenge to face diseases of great impact, such as cancer or infectious diseases where current treatments exhibit some major limitations mostly related to their poor pharmacokinetic profile [11]. Indeed, the development of a specific drug delivery device able to increase drug efficacy reducing side-effects could be useful to improve patient compliance. Protein-based nanoparticles possess other peculiar characteristics depending on the type of protein 
that constitutes the scaffold, which could find application in clinics. Among protein nanocages we can include human protein such as Vault particles, Heat Shock Proteins and ferritins, but also viral protein like those which form the viral capsid [12].

The latter are constituted by one many viral capsid proteins. Although they include a wide variety of sizes and morphologies with well-defined geometries (icosahedral or helical), capsids possess uniform shape and robust protein shell, suitable for biological and chemical conjugation and stable over a wide range of $\mathrm{pH}$ and temperatures [1, 8]. Despite virus particles have found wide application in gene therapy and vaccines, they have been also used as drug delivery devices [8]. A virus particle functionalized with folic acid has been used to mediate the delivery of doxorubicin to ovary cancer cells with a significant increase in drug cellular uptake and subsequent higher efficacy [1]. Neverthless viral protein are quite difficult to produce in bulk strongly limiting their biomedical application, and some issues about the host immunological response still have to be addressed [1, 13].

Vault particles represent an extremely interesting and versatile solution with high potential both as drug delivery device, both as imaging agent because: 1) they have a spacious internal volume, which is suitable for the encapsulation of bioactive molecules; 2) they are constituted by naturally occurring amino-acids sequences with no declared immunogenicity and toxicity; 3) they are easy to genetically modify; and 4) they are arranged in a dynamic cage that can disassemble into two halves in response to a lowering of the $\mathrm{pH}[14]$. At present, some studies have been performed using this particle as drug delivery device, while have not yet found application as imaging agents [14].

Heat Shock Protein (sHsp) has the external size of sHsp is $12 \mathrm{~nm}$ in diameter and is constituted by 24 subunits arranged to outline the internal cavity of $6.5 \mathrm{~nm}$ in diameter. The sHsp architecture displays large size pores of about $3 \mathrm{~nm}$ in diameter able to mediate the free exchange of cargo between the inside and the outside [1]. As other caged proteins, it is stable until $70{ }^{\circ} \mathrm{C}$ and in a $\mathrm{pH}$ range between 5 to 11 , implying its resistance to the harsh conditions under which chemical 
modifications take place [15]. Moreover, since the sHsp cage does not contain cysteine residues, they could be introduced by site-directed mutagenesis to allow further attachment of bioactive molecules [15]. Also, lysine residues have been used for chemical conjugation, while the genetic fusion with melanoma and lymphocyte cell-specific targeting moieties has been exploited to deliver doxorubicin [16]. But in our opinion, the most promising protein based-nanocage is represented by ferritin. Ferritin nanoparticles can be easily produced by DNA recombinant technology in host organisms with good yields [17]. They possess a $8 \mathrm{~nm}$ central cavity loadable with transition metals, drugs, fluorescent molecules or other contrast agents with high efficiency [18]. Moreover, the protein shell of ferritins has been be easily modified via either chemical or genetic modifications to introduce functionalities, such as targeting moieties, drugs, quenchers, dyes, contrast agents and even siRNAs [19]. Ferritin nanocages constituted by 24-mer of $\mathrm{H}$ subunits have already been largely explored for cytotoxic drug delivery into cancer sites with encouraging results [20]. The natural tumor homing of $\mathrm{H}$-ferritin nanocages due to specific recognition of the transferrin receptor 1 (TfR-1), which is overexpressed several cancer subtypes, ensured the high efficacy otained with these nanoparticles [20]. Ferritin have also been reported as a good nanovector for nuclear delivery of drug exploiting a physiological mechanism of nuclear translocation [17]. In this case, a Doxorubicin-loaded H-Ferritin is able to mediate DOX self-triggered nuclear translocation increasing drug efficacy. The sum of all these data makes ferritin the most appealing protein nanocage, and also the candidate of choice for the future applications in the field of nanomedicine. Indeed, a lot of work has already been done for the development of ferritin-based devices for MRI, optical imaging, hyperthermal therapy and drug delivery. As mentioned above, promising results have been obtained allowing the development of multifunctional devices for imaging and therapy. Despite this, much work has to be done to enhance the knowledge about (1) the fate of ferritin and its derivatives after systemic injection, (2) their toxicity in off-target organs, (3) the real impact if immunogenicity and toxicity of ferritin derivatives for further clinical translation. Although further research is still strongly required, ferritin nanoparticles are undoubtedly the protein-based 
nanocages with the translational highest potential for development of smart imaging and therapeutic tools in the next future.

\section{REFERENCES}

[1] Maham A, Tang Z, Wu H, Wang J, Lin Y. Protein-based nanomedicine platforms for drug delivery. Small, 5, 1706-1721 (2009)

[2] Li SD, Huang L. Pharmacokinetics and biodistribution of nanoparticles. Mol Pharm, 5, 496-504 (2008).

[3] Peer D, Karp JM, Hong S, Farokhzad OC, Margalit R, Langer R. Nanocarriers as an emerging platform for cancer therapy. Nat Nanotechnol, 2,751-760 (2007)

[4] Mok H, Zhang M. Superparamagnetic iron oxide nanoparticle-based delivery systems for biotherapeutics. Expert Opin Drug Deliv 10, 73-87 (2013).

[5] De Jong WH, Borm PJ. Drug delivery and nanoparticles: applications and hazards. Int J Nanomedicine, 3, 133-149 (2008).

[6] Molino NM, Wang S-W. Caged protein nanoparticles for drug delivery. Current Opinion in Biotechnology, 28, 75-82 (2014).

[7] Elzoghby AO, Samy WM, Elgindy NA. Protein-based nanocarriers as promising drug and gene delivery systems. J Control Release, 161, 38-49 (2012).

[8] Stanley S. Biological nanoparticles and their influence on organisms. Current Opinion in Biotechnology, 28, 69-74 (2014).

[9] Lim S. Protein Nanocages. The versatile molecular shell. Asia-Pacific Biotech News, 17, 51-53 (2013).

[10] Reichen J. The Role of the Sinusoidal Endothelium in Liver Function. News Physiol. Sci. 14, 117- 121 (1999).

[11] Yewale C, $\underline{\text { Baradia } \quad \mathrm{D}}$, Vhora I, Misra A. Proteins: emerging carrier for delivery of cancer therapeutics. Expert Opin Drug Deliv 10, 1429-48 (2013). 
[12] Hecht S. Construction with Macromolecules. Mater Today, 8, 48-55 (2005).

[13] Zeltins A. Construction and characterization of virus-like particles: a review. Mol. Biotechnol., 53, 92-107 (2013).

[14] Rome LH, Kickhoefer V. Development of the Vault Particle as a Platform Technology. ACS Nano, 7, 889-902 (2013).

[15] Flenniken MW, Willits DA, Brumfield S, Young MJ, Douglas T. The Small Heat Shock Protein Cage from Methanococcus jannaschii Is a Versatile Nanoscale Platform for Genetic and Chemical Modification. Nano Lett., 3, 1573-1576 (2003)

[16] Flenniken ML, Liepold LO, Crowley BE, Willits DA, Young MJ, Douglas T. Selective attachment and release of a chemotherapeutic agent from the interior of a protein cage architecture. Chem Commun, 447-449 (2005)

[17] Bellini M, Mazzucchelli S, Galbiati E, Sommaruga S, Fiandra L, Truffi M, Rizzuto MA, Colombo M, Tortora P, Corsi F, Prosperi D. Protein nanocages for self-triggered nuclear delivery of DNA-targeted chemotherapeutics in Cancer Cells. J Control Release, 196, 184-196 (2014).

[18] Zhen Z, Tang W, Guo C, Chen H, Lin X, Liu G, Fei B, Chen X, Xu B, Xie J. Ferritin nanocages to encapsulate and deliver photosensitizers for efficient photodynamic therapy against cancer. ACS Nano, 7, 6988-6996 (2013).

[19] Lin X, Xie J, Niu G, Zhang F, Gao H, Yang M, Quan Q, Aronova MA, Zhang G, Lee S, Leapman R, Chen X. Chimeric ferritin nanocages for multiple function loading and multimodal imaging. Nano Lett., 11, 814-19 (2011).

[20] Liang M, Fan K, Zhou M, Duan D, Zheng J, Yang D, Feng J, Yan X. H-ferritin-nanocaged doxorubicin nanoparticles specifically target and kill tumors with a single-dose injection. $\underline{\text { Proc Natl }}$ Acad Sci U S A. 111, 14900-5 (2014). 